\title{
Juno Therapeutics
}

National Cancer Institute

\section{Source}

National Cancer Institute. Juno Therapeutics. NCI Thesaurus. Code C126404.

A clinical-stage company developing cellular immunotherapies based on Chimeric Antigen Receptors (CARs) and T Cell Receptors (TCRs) technologies. 